\title{
Molecular Docking Study of Cassia tora, Brassica campestris and Calotropis procera as Acetylcholinesterase Inhibitor
}

\author{
Malabade Rohit', Taranalli Ashok, Rasal Vijaykumar and Kamlesh Kashniyal \\ Depatment of Pharmacology, KLE University's college of Pharmacy, Belagavi, Karnataka, India.
}

\begin{abstract}
Introduction: Acetylcholinesterase inhibitor is the only standard and FDA approved drug therapy for Alzheimer's disease and its associated disorders. Numerous plants and their phytoconstiuents are being reported to inhibit acetylcholinesterase. To get insight of the intermolecular interactions, the molecular docking studies are performed at active site of acetylcholinesterase enzyme. Aim: In this study, an attempt is made for identification of potential ligands from selected 30 compounds which are reported to be present in Cassia tora, Brassica campestris and Calotropis procera, targeted against acetylcholinesterase using molecular modelling and docking studies. Results: The relative binding affinity of the compounds towards AChE was selected on the basis of docking score, GLIDE score and interaction patterns.Several compounds showed stronghydrogen bonding to several important amino acid residues andtheir hydrophobic interactions could also explain their potency to inhibit acetylcholinesterase. These compoundsbelong to different classes like flavonoids, vitamin, cardenolides and etc. Some of these compounds have been reported for their beneficial effect on dementia related disorders, while remaining are suggested to be potential acetylcholinesterase inhibitors. Conclusion: Hence, this study provides evidencefor consideration of valuable ligand molecule as potential acetylcholinesterase inhibitorandfurther in vitro and in vivo investigations may prove its therapeutic potential
\end{abstract}

Key words: Alzheimer's disease, Molecular Docking, Phytoconstituent, Virtual screening, Cognition enhancer.

\section{INTRODUCTION}

Alzheimer's disease is the leading cause of dementia, accounting for about $50 \%$ of all cases worldwide. ${ }^{1}$ It is characterized by progressive loss of memory and extracellular deposition of amyloid- $\beta$ peptides and intracellular neurofibrillary tangles. ${ }^{2}$ There has been remarkable improvement in understanding of the pathogenesis of Alzheimer's disease in last few decades. Several independent hypotheses like oxidative stress, dysfunction of cholinergic transmission, inflammatory reaction and Amyloid $\beta$ metabolism has been put forward but still the exact mechanism remains unclear. ${ }^{3}$

'Cholinergic hypothesis' is one of the important hypotheses. Also, inhibition of acetylcholinesterase serves as promising strategy for the treatment of Alzheimer's disease, senile dementia, ataxia, myasthenia gravis and Parkinson's disease. ${ }^{4}$ Synthetic drugs approved for treating Alzheimer's disease are tacrine and donepezil, the naturally derived drugs are rivastigmine, physostigmine but these compounds have been reported to have adverse effects like gastrointestinal disturbances and problems associated with bioavailability which necessitates the interest in finding better $\mathrm{AChE}$ inhibitors from natural resources. ${ }^{5}$ Although, some natural products have shown potential to enhance the cholinergic function in CNS by cholinesterase inhibition, but problem persists with target selection due to chemical diversity of natural products. ${ }^{6}$ To overcome this ambiguity in effect, in silico molecular docking approach is adopted, which can be helpful in identifying lead compound with potent inhibitory activity
Submission Date : 09-06-2015 Revision Date : :13-08-2015 Accepted Date : :24-08-2015

DOI: 10.5530/ijper.50.1.15 Correspondence Address Mr. Rohit Malabade PhD Research Scholar, Depatment of Pharmacology KLE University's college of Pharmacy, Belagavi, Karnataka, India. Email:malabade_rohit@ yahoo.co.in

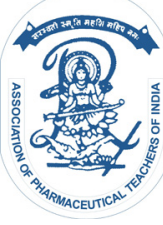

www.ijper.org 


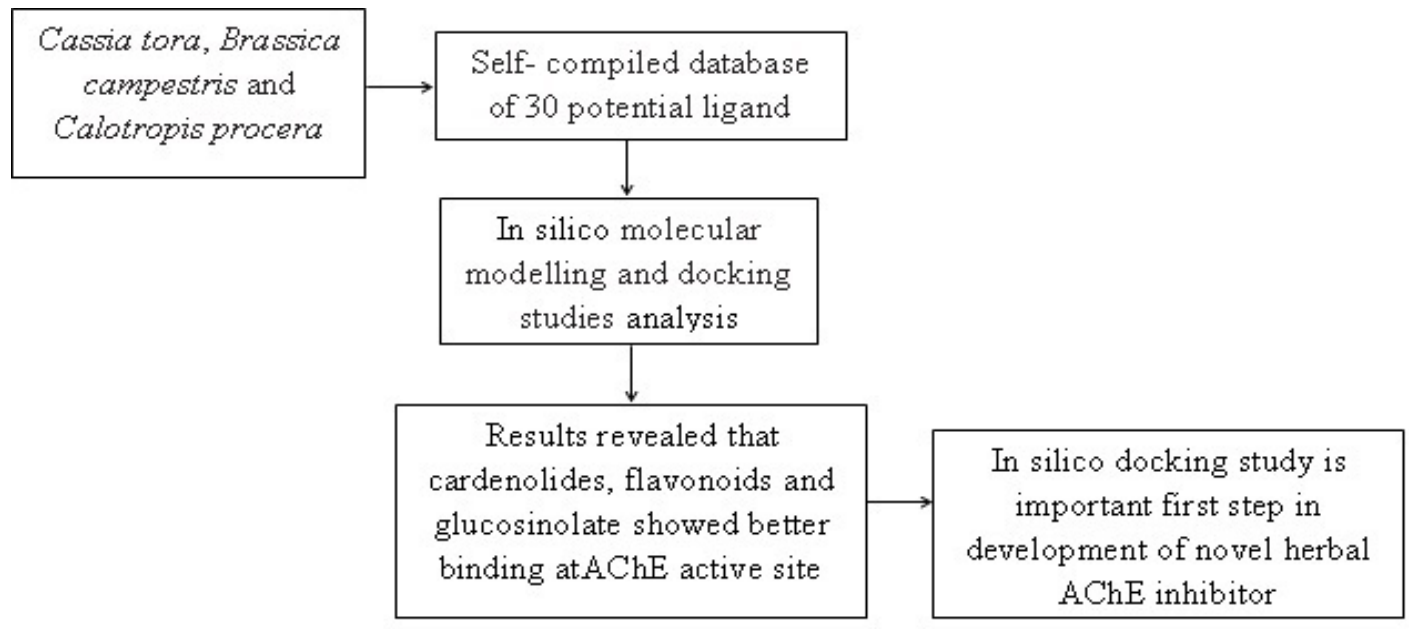

\section{Graphical Abstract}

\begin{tabular}{|c|c|c|c|}
\hline Plant & Part of Plant & Chemical class & Chemical constituent \\
\hline \multirow{12}{*}{ Calotropis procera $(\mathrm{CP})$} & \multirow{12}{*}{ Latex } & \multirow{10}{*}{ Cardenolides } & 1. Calotropin \\
\hline & & & 2. Calotoxin \\
\hline & & & 3. Calactin \\
\hline & & & 4. Uscharin \\
\hline & & & 5. Voruscharin \\
\hline & & & 6. Uzarigenin \\
\hline & & & 7. Syriogenin \\
\hline & & & 8. Proceroside \\
\hline & & & 9. Calotropagenin \\
\hline & & & 10. $\beta$-amyrin \\
\hline & & Terpenes & 11. Lupeol \\
\hline & & Flavanoid & 12. Quercetin-3- rutinoside \\
\hline \multirow{12}{*}{ Brassica Campestris (BC) } & \multirow{12}{*}{ Seed } & \multirow{3}{*}{ Phytosterol } & 1. Brassicasterol \\
\hline & & & 2. Campesterol \\
\hline & & & 3. Sitosterol \\
\hline & & \multirow{7}{*}{ Glucosinolate } & 4. Sinigrin \\
\hline & & & 5. Gluconapine \\
\hline & & & 6. Glucobrassicanapin \\
\hline & & & 7. Sinalbin \\
\hline & & & 8. Glucobrassicin \\
\hline & & & 9. Neoglucobrassicin \\
\hline & & & 10. 4-hydroxy glucobrassicin \\
\hline & & Phenolic compound & 11. Sinapine \\
\hline & & Vitamin & 12. Vitamin $A$ \\
\hline \multirow{6}{*}{ Cassia tora $(\mathrm{CT})$} & \multirow{6}{*}{ Leaves } & \multirow{2}{*}{ Anthraquinone } & 1. Emodin \\
\hline & & & 2. Physcion \\
\hline & & \multirow{4}{*}{ Flavonoid } & 3. Quercitin \\
\hline & & & 4. Uridine \\
\hline & & & 5. Stigmasterol \\
\hline & & & 6. $\beta$ stigmaterol \\
\hline
\end{tabular}




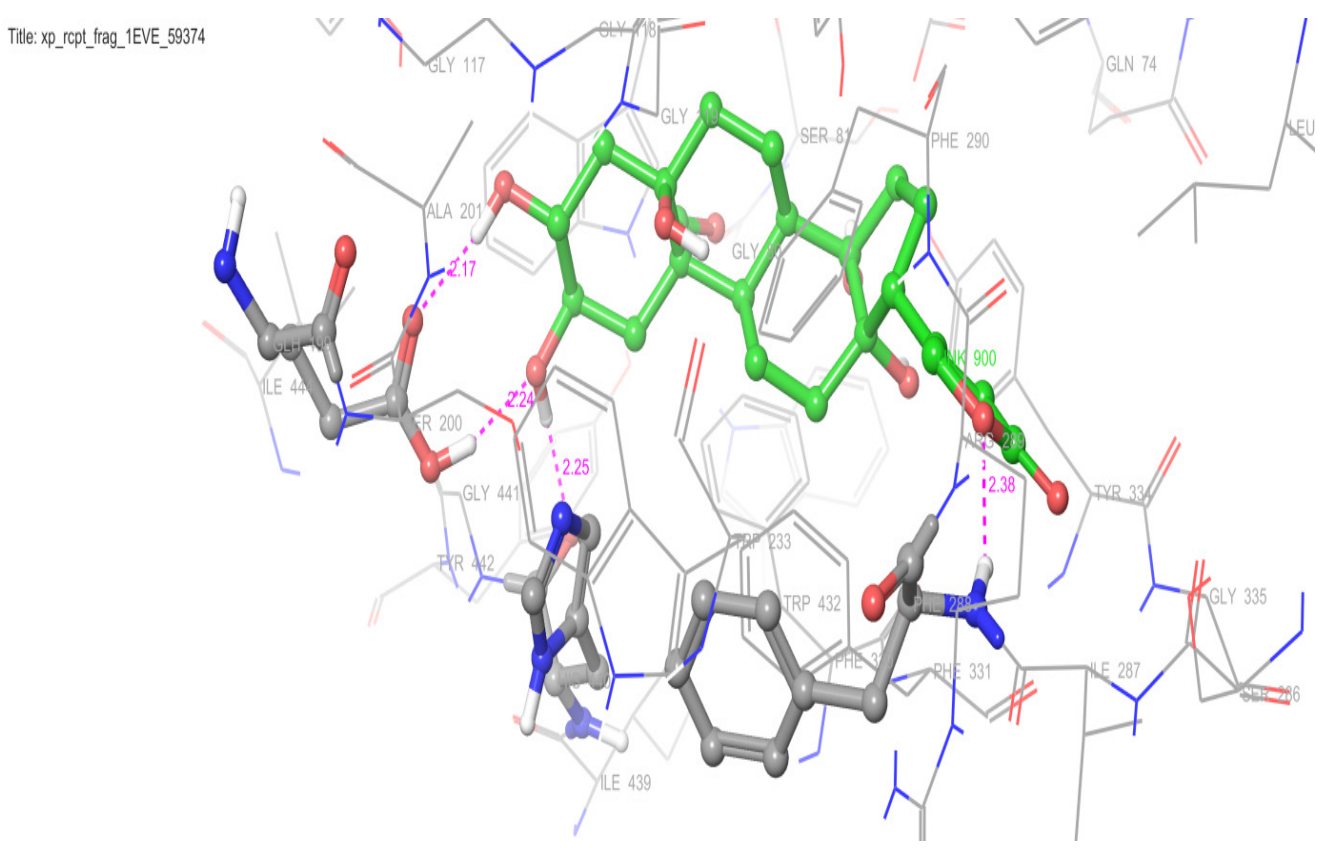

Figure 1A: Orientation of Calotropagenin from Calotropis procera in 1EVE active pocket

The $\mathrm{O}$ of oxane group of compound forms hydrogen bond with PHE288 (O---NH, distance: 2.38Å), $\mathrm{H}$ and $\mathrm{O}$ of hydroxyl group of compound forms hydrogen bond with HIS $440(\mathrm{OH}---\mathrm{N}$, distance: $2.25 \AA$ $)$ and GLH $199(\mathrm{O}---\mathrm{OH}$, distance: $2.24 \AA \AA$ ) respectively, and $\mathrm{H}$ of hydroxyl group forms hydrogen bond with GLH199 $(\mathrm{H}---\mathrm{O}$, distance: $2.17 \AA \AA)$.
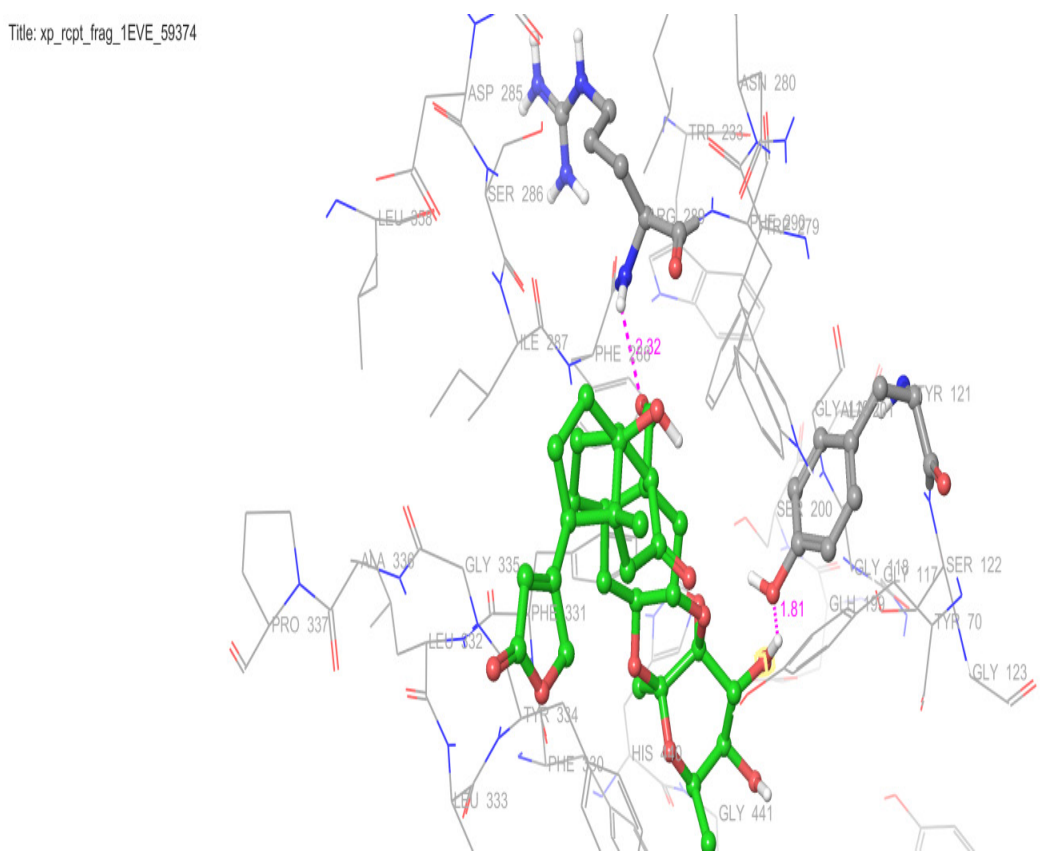

Figure 1B: Orientation of Calotoxin from Calotropis procera in 1EVE active pocket

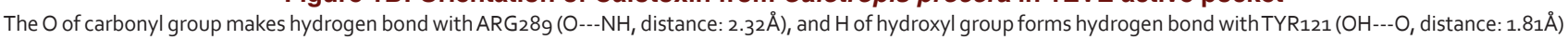

and also helps to understand the insight of interaction of compounds with target receptor or protein. ${ }^{7}$

In search for natural products with AChE inhibitor activity, this study is focused on in silico molecular docking studies to explore the ability of reported compounds from Cassia tora, Brassica campestris and Calotropis procera latex to potentially inhibit AChE. This was achieved through the analysis of information of the reported compounds from available literature sources and other information databases. The selected three plants have been proved scientifically for the treatment of various diseases including CNS disorders, but interaction of their phytoconstituent with AChE receptor is not well understood, hence Cassia tora, Brassica campestris and Calotropis procera was selected for study. Also, this study attempts to focus on the discovery of novel molecules from Cassia tora, Brassica campestris and Calotropis procera latex that can bind and inhibit AChE enzyme activity. 
Tite: xp_rcpt_trag_1EVE_-42555



Figure 2A: Orientation of Vitamin A from Brassica campestris in 1EVE active pocket

The $\mathrm{H}$ of hydroxyl group makes hydrogen bond with $\mathrm{HIS} 44 \mathrm{O}(\mathrm{OH}---\mathrm{N}$, distance: $2.61 \AA)$ and SER200 $(\mathrm{OH}---\mathrm{O}$, distance: $2.54 \AA)$, and $\mathrm{H}$ of hydroxyl group forms hydrogen bond with SER200 (OH---O, distance: $2.10 \AA)$.

Titte: XP_crot_frag_1EVE__42555

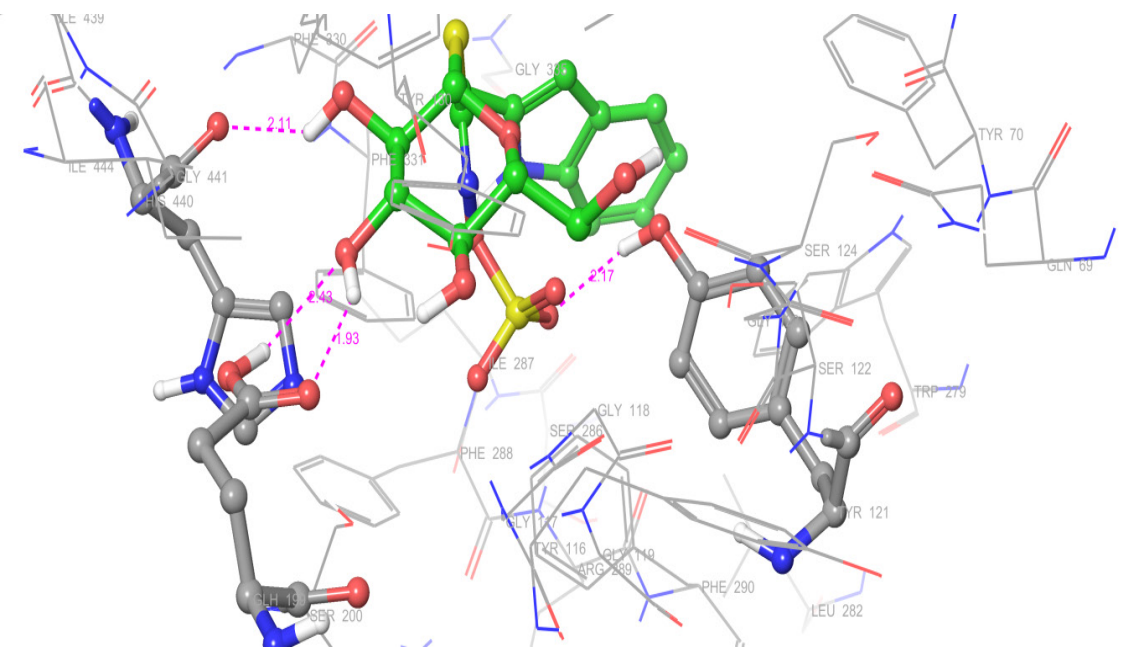

Figure 2B: Orientation of Glucobrassicin from Brassica campestris in 1EVE active pocket

The $\mathrm{H}$ of hydroxyl group forms hydrogen bond with HIS $440(\mathrm{OH}---\mathrm{O}$, distance: $2.11 \AA \AA)$, $\mathrm{H}$ and O of hydroxyl group makes hydrogen bond with $\mathrm{GLH} 199(\mathrm{OH}---\mathrm{O}, \mathrm{O}---\mathrm{OH}$; distance: $1.93 \AA$ and $2.43 \AA$ respectively), and $\mathrm{H}$ of thio group forms hydrogen bond with TYR $121(\mathrm{SH}---\mathrm{OH}$, distance: $2.17 \AA)$.

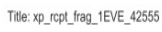

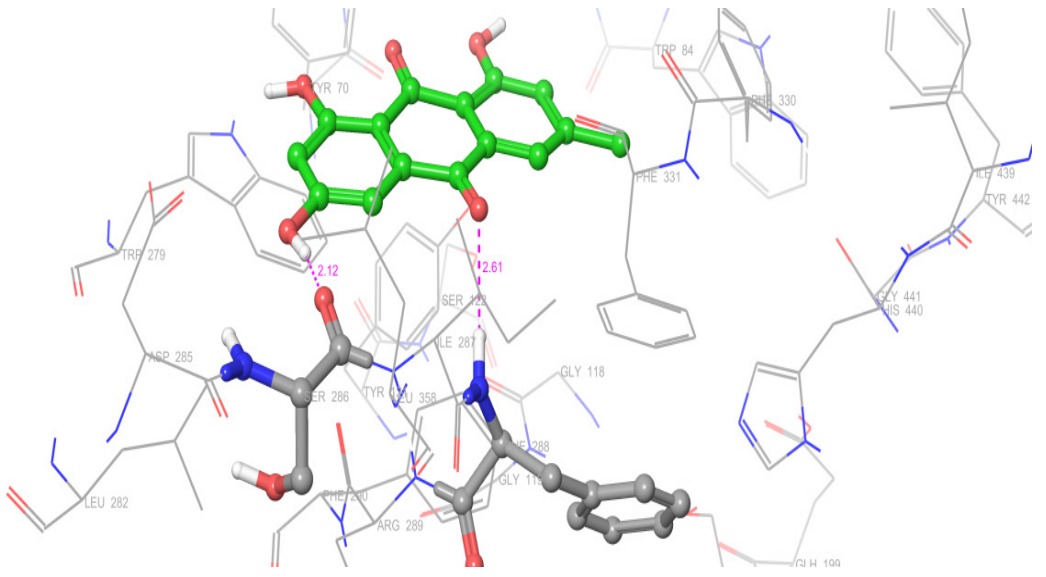

Figure 3: Orientation of physocin from Cassia tora in 1EVE active pocket

The $\mathrm{H}$ of hydroxyl group of our compound makes hydrogen bond with SER286 ( $\mathrm{H---O}$, distance: $2.12 \AA)$, and carbonyl oxygen forms hydrogen bond with PHE288 (O---NH, distance: $2.62 \AA$ ) 


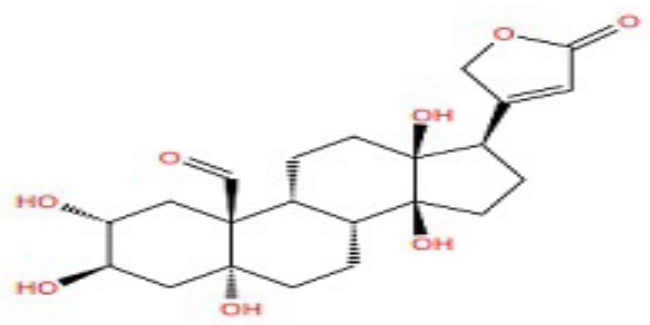

(a)

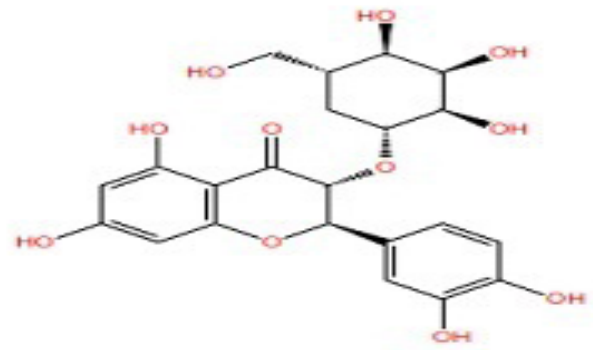

(c)

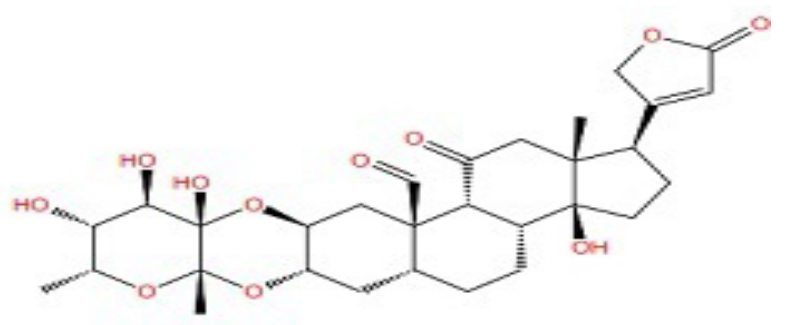

(b)

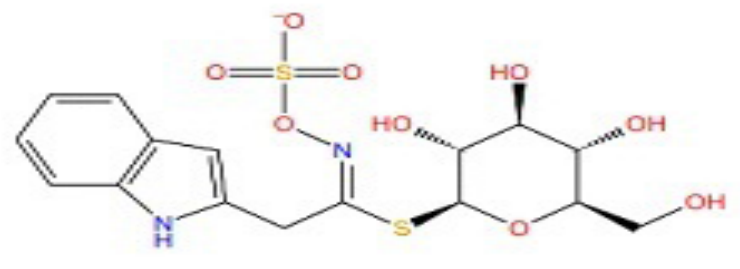

(d)<smiles>Cc1cc(O)c2c(c1)C(=O)c1cc(O)cc(O)c1C2=O</smiles>

(e)

Figure 4: Structure of most active compounds from Cassia tora, Calotropis procera and Brassica campestris (a-Calotropagenin, b-Calotoxin, c-Vitamin A, d-Glucobrassicin, e-Physocin).

\section{MATERIALS AND METHODS}

\section{Protein structure preparation}

3D structure of acetylcholinesterase complexed with Aricept (PBD ID: 1EVE) was retrieved from RCSB Protein Data Bank (PDB). The major form of Torpedo calfornica (Tc) AChE is a homodimer, covalently attached to plasma membrane through phosphatidylinositol group. It has ellipsoidal shape and belongs to the class of $\alpha / \beta$ proteins and consists of a 12 stranded central mixed $\beta$ sheet surrounded by $14 \alpha$ helices, it also contains two active subsites, esteratic and anionic. ${ }^{8}$ The E2020 has a unique orientation, extending from the anionic subsite of the active site to the peripheral anionic site. The E2020 makes principal interactions along the active subsites of the enzyme through the benzyl moiety, the piperidine nitrogen, and the dimethoxyindanone moiety. ${ }^{9}$ The obtained protein structure was prepared for molecular docking by removal of water molecules from its 3D structure. To depict the in vivo interaction, the energy of the target protein was minimized before performing the docking simulations.

\section{Ligand structure preparation}

In this study, overall 30 compounds reported to be present in leaves of Cassia tora ${ }^{10}$, latex of Calotropis procera ${ }^{11}$ and seeds of Brassica campestris ${ }^{12}$ which are of medicinal importance were selected from the literature survey (Table 1). These comprise diverse series of compounds including flavonoids, cardenoloids, steroids, terpenes, vitamins, phenols etc. For the development of pharmacophore, the 2D structures of the compounds identified were imported into Discovery Studio (DS) version 2.1 (Discovery Studio, 2012) and then converted into $3 \mathrm{D}$ structures, so that molecules can align in energetically favourable conformation to bind to a receptor site.

\section{Docking protocol}

To study the molecular basis of interaction and affinity for binding of selected compounds to AChE protein, all the ligands were docked into the active site of AChE. 
The structural binding pockets and active sites in the protein were determined by literature search.

The molecular docking studies were performed using Maestro, Schrodinger suite. The best docked structure was chosen using a GLIDE score (Dock Score) function and Hydrogen bonding. GLIDE score incorporates the coulomb and vdW interaction energies between the ligand and the receptor and also introduces a solvation model. It has negative regression coefficient towards the binding affinity. GLIDE module was set to extra precision (XP) mode so that Hydrogen bond could be visualized in the XP Visualizer. ${ }^{13}$

\section{RESULT AND DISCUSSION}

The main objective of this study is to identify novel acetylcholinesterase inhibitors based on their interaction with AChE. The protocol was validated by reproducing conformation of docked ligand in crystal structure of $\mathrm{AChE}$ and the docked Aricept was removed from active site and docked again. The top three conformations of Aricept were taken into consideration. The acceptable result denotes that the docked ligand is having similar binding position and orientation with that of Aricept.

In the present study, preliminary screening of 30 chemically diverse compounds was carried out for their inhibitory effect on AChE. Calotropagenin (cardenolides) present in latex of Calotropis procera showed significant GLIDE score of -13.02 and bond strength of $-4.23 \AA$ (Figure 1A and 4) as compared to GLIDE score of -11.78 and bond strength of $-1.27 \AA$ for AriceptAChE complex. While, Calotoxin has GLIDE score of -11.23 and bond strength of $-2.51 \AA$ (Figure $1 \mathrm{~B}$ and 4), also Quercetin-3-rutinoside has GLIDE score of -11.21 and bond strength of $-0.66 \AA$ showed interaction comparable with Aricept-AChE complex. This binding study may predict mode of action and active chemical moiety from Calotropis procera latex responsible for AChE inhibition and its claim to possesses anti-amnesic ${ }^{14}$, anti-convulsant ${ }^{15}$ and neuroprotective effects. ${ }^{16}$

Among the selected compounds from seeds of Brassica campestris, Vitamin A showed GLIDE score -11.02 and bond strength $-5.54 \AA$ (Figure $2 \mathrm{~A}$ and 4) while Glucobrassicin (Glucosinolate) has GLIDE score of -10.85 and bond strength of $-4.0 \AA$ (Figure 2B and 4) which denotes tight binding and high energies when compared with Aricept- $\mathrm{AChE}$ complex. This result indicate the beneficial effect of Vitamin A in cognition enhancing effect and is currently being studied for its beneficial effect on Alzheimer's disease. ${ }^{17}$ Also, the presence of Glucosinolate in higher concentration in Brassica campestris species can be responsible for its cognition enhancement activity. ${ }^{18}$

While compounds like physcion from Cassia tora leaves has GLIDE score of -10.2 and bond strength of -2.22 $\AA$ (Figure 3 and 4) showed tight binding between ligand and $\mathrm{AChE}$, the flavonoids like quercetin, sigmasterol, $\beta$ stigmasterol showed comparable GLIDE score but weak bonding with AChE proteins when compared with Aricept-AChE complex. These results are in close proximity with earlier studies reporting beneficial effect of flavonoid in CNS disorders ${ }^{19}$, also proves the use of Cassia tora as nervine tonic and neuroprotective agent. ${ }^{20}$

\section{CONCLUSION}

A structure-based pharmacophore model was generated and validated to obtain active AChE inhibitors from our self-compiled database of known compounds reported from leaves of Cassia tora, latex of Calotropis procera and seeds of Brassica campestris. Also an attempt was made to co-relate their reported biological activity along with marketed AChE inhibitors with their docking scores.

The docking study revealed that cardenolides, flavonoids and glucosinolate showed better alignment at active site by interacting with all crucial amino acid residues. Thus, the in silico method adopted in the present study helped in identifying the lead compounds and also may partly explain their beneficial effect in in vivo study. Overall, this could be an important first step in development of novel AChE inhibitor. Studies have been undertaken to isolate the compounds and to test their effect in in vitro assays and in in vivo models.

\section{ACKNOWLEDGEMENT}

The author is thankful to KLE College of Pharmacy, Belagavi, Karnataka, India for providing the facilities to carry out the study.

\section{ABBREVIATION}

AChE : Acetylcholinesterase

GLIDE: Grid based Ligand Docking with Energetics

AD : Alzheimer's disease

CNS : Central nervous system

FDA : Food and drug Administration 


\section{SUMMARY}

- 30 compounds which are of medicinal importance and arer eported to be present in leaves of Cassia tora, latex of Calotropis procera and seeds of Brassica campestris were selected from the literature survey.

- These compound was docked with 3D structure of acetylcholinesterase complexed with Aricept (PBD ID: 1EVE) retrieved from RCSB Protein Data Bank.

- The docking study results revealed that cardenolides, flavonoids and glucosinolate showed better alignment at active site by interacting with all crucial amino acid residues.

\section{About Authors}

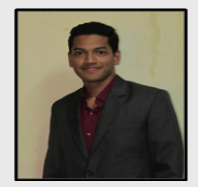

Mr Rohit Malabade: Is a doctoral student at the KLE University, Belagavi, where he graduated in Master of Pharmacy. His doctoral research is focused on in vivo and in vitro evaluation of selected medicinal plant for cognition enhancing activity.

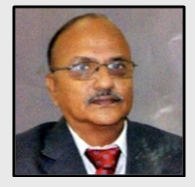

Dr. Ashok Taranalli: Is currently Dean and Professor in department of pharmacology at KLE College of Pharmacy, Belagavi. $\mathrm{He}$ has experience in the areas of Pharmacology and Chemistry of Natural

\section{REFERENCES}

1. Suzhen Dong, Yale Duan, Yinghe Hu, Zheng Zhao. Advances in the pathogenesis of Alzheimer's disease: a re-evaluation of amyloid cascade hypothesis. Translational Neurodegeneration 2012; 1(18):1-12.

2. Froestl W, Pfeifer A, Muhs A. Cognitive enhancers (Nootropic) Part 3 Drug interacting with targets other than receptor or enzymes disease modifying drugs. J Alz Dis 2013; 34:1-114.

3. Nicolakakis N, Hamel E. Neurovascular function in Alzheimer's disease patients and experimental models. Journal of Cerebral Blood Flow \& Metabolism (2011) 31, 1354-70.

4. Agrawal R, Tyagi E, Saxena G, Nath C.Cholinergic influence on memory stages: A study on scopolamine amnesic mice. Indian J Pharmacol 2009; 41(4):192-6.

5. Mukherjee P, Kumar V, Mal M, Houghton P. Acetylcholinesterase inhibitors from plants. Phytomedicine 2007; 14:289-300.

6. Rasool $\mathrm{M}$ et al. Recent Updates in the Treatment of Neurodegenerative Disorders Using Natural Compounds. Evidence-Based Complementary and Alternative Medicine 2014:1-7.

7. Greco I et al. Alzheimer's disease biomarker discovery using in silico literature mining and clinical validation. Journal of Translational Medicine 2012; 10(1):1-10.

8. Sussman J, Harel M, Frolow F, Oefner C, Silman I. Atomic structure of Acetylcholinesterase from Torpedo calfornica: a prototypic Acetylcholinebinding protein. Science 1991; 253(5022):872-79.

9. Kryger G, Silman I, Sussman J. Structure of acetylcholinesterase complexed with E2020 (Aricept): implications for the design of new anti-Alzheimer drugs. Structure 1999; 7(3):297-307.

10. Jain S, Patil U K. Phytochemical and Pharmacological profile of Cassia tora Linn - an overview 2010; 1(4):430-7.

Products, his work is focused in Medicinal plants, New Research Drugs and CNS disorders.

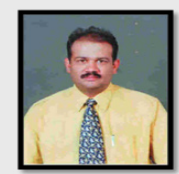

Dr. V. P. Rasal: Is currently Principal at KLE College of Pharmacy, Belagavi. He has experience in the areas of Pharmacology and Toxicology.
11. Ranjit P, Rao E, Krishnapriya M, Nagalakshmi V, Silpa P. An overview of phytochemical and pharmacological activities of calotropis procera. FS $\mathrm{J}$ Pharm Res 2012; 1(2):18-25.

12. Kumar V, Thakur A, Barothia D, Chatterjee S. Therapeutic potentials of Brassica juncea: an overview. Int J Gen Trad Med 2011; 1(1):1-17.

13. Halperin I, Ma B, Wolfson H, Nussinov R. Principles of Docking: An Overview of Search Algorithms and a Guide to Scoring Functions. PROTEINS: St Fun Gen. 2002; 47:409-43

14. Gavimath C, Havannavar V, Hulekal P, Pattar P, Joshi H. Evaluation of Antiamnesic Potentials of Calotropis Procera in Mice. Pharmacologyonline 2009; 3:457-62.

15. Jalalpure S S. Anticonvulsant effects of Calotropis procera root in rats. Pharm Bio 2009; 47(2):162-7.

16. Kumar A, Yadav A, Rao M. Ayurvedic uses and pharmacological activities of Calotropis procera Linn. Asian J Trad Med 2011; 6(2):45-53.

17. Sugimoto $H$, Yamanishi $Y$, Limura $Y$, Kawakami Y. Donepezil Hydrochloride (E2020) and Other Acetylcholinesterase Inhibitors. Current Medicinal Chemistry 2000; 7(3):303-39

18. Zukalova H, Vasak J. Role and effect of Glucosinolates of Brassica species-a review. ROSTLINNÁ VÝROBA, 48, 2002 (4):175-180.

19. Tareq H, Orhan I, Kartal M, Dvorská M. Cholinesterase inhibitory activities of some flavonoid derivatives and chosen xanthone and their molecular docking studies. Chemico-Biological Interactions 2009; 181(3):383-9.

20. Xu-zhao Li, Shuai-nan Zhang, Shu-min Liu, Fang Lu. Recent advances in herbal medicines treating Parkinson's disease. Fitoterapia 2013; 84(1):273-85. 